\title{
Extended Renal Outcomes with Use of Iodixanol versus Iohexol after Coronary Angiography
}

\author{
Horng-Ruey Chua, ${ }^{1,2}$ Mark Horrigan, ${ }^{3}$ Elizabeth Mcintosh, ${ }^{3}$ and Rinaldo Bellomo ${ }^{1,4}$ \\ ${ }^{1}$ Department of Intensive Care, Austin Hospital, Melbourne, VIC 3084, Australia \\ ${ }^{2}$ Division of Nephrology, University Medicine Cluster, National University Hospital, National University Health System, \\ Singapore 119228 \\ ${ }^{3}$ Department of Cardiology, Austin Hospital, Melbourne, VIC 3084, Australia \\ ${ }^{4}$ Australian and New Zealand Intensive Care Research Committee (ANZIC-RC), School of Public Health and Preventive Medicine, \\ Monash University, Melbourne, VIC 3181, Australia \\ Correspondence should be addressed to Rinaldo Bellomo; rinaldo.bellomo@austin.org.au
}

Received 10 February 2014; Revised 27 June 2014; Accepted 21 July 2014; Published 7 August 2014

Academic Editor: Alejandro Ferreiro

Copyright @ 2014 Horng-Ruey Chua et al. This is an open access article distributed under the Creative Commons Attribution License, which permits unrestricted use, distribution, and reproduction in any medium, provided the original work is properly cited.

\begin{abstract}
The impact of isoosmolar versus low-osmolar contrast media (CM) administration on contrast-induced acute kidney injury (CI-AKI) and extended renal dysfunction (ERD) is unclear. We retrospectively examined incidences of CI-AKI and ERD in patients who received iodixanol (isoosmolar) versus iohexol (low-osmolar) during angiography for cardiac indications. Of 713 patients, 560 (cohort A), 190 (cohort B), and 172 (cohort C) had serum creatinine monitored at 3 days, 30 days, and 6 months after angiography, respectively. $18 \%$ of cohort A developed CI-AKI, which was more common with iodixanol than iohexol (22\% versus $13 \%, P=0.006)$. However, patients given iodixanol were older with lower baseline estimated glomerular filtration rates (eGFR). On multivariate analysis, independent associations with higher CI-AKI risk include age $>65$ years, female gender, cardiac failure, ST-elevation myocardial infarction, intra-aortic balloon pump, and critical illness, but not CM type, higher CM load, or eGFR $<45 \mathrm{~mL} / \mathrm{min} / 1.73 \mathrm{~m}^{2} .32 \%$ of cohort B and $34 \%$ of cohort C had ERD at 30 days and 6 months, while $44 \%$ and $41 \%$ of subcohorts had ERD at 90 days and 1 year, respectively. CI-AKI, but not CM type, was associated with medium- and longer-term ERD, with 3-fold higher risk. Advanced age, emergent cardiac conditions, and critical illness are stronger predictors of CI-AKI, compared with CM-related factors. CI-AKI predicts longer-term ERD.
\end{abstract}

\section{Introduction}

Contrast media $(\mathrm{CM})$ is the third most common cause of hospital-acquired acute kidney injury (AKI), and coronary angioplasty accounts for the highest incidence of contrastinduced AKI (CI-AKI) [1]. CI-AKI risk is exacerbated by comorbidities including advanced age, diabetes mellitus $(\mathrm{DM})$, congestive cardiac failure (CCF), and chronic kidney disease (CKD) [2], all of which are highly prevalent in patients with coronary artery disease. Thus, numerous CI-AKI preventive strategies have been employed, such as reduced CM load and avoidance of recurrent exposure [3], intravascular volume expansion [4], N-acetylcysteine administration [5], and preferred use of isoosmolar CM (IOCM) or low-osmolar CM (LOCM) over high-osmolar CM (HOCM) [6].

In relation to $\mathrm{CM}$, use of IOCM iodixanol has been reported to reduce CI-AKI risk in patients with DM and CKD, compared to LOCMs [7], in particular iohexol [8]. Therefore, clinicians may prefer to use iodixanol in high-risk subjects. However, findings on the apparent benefit of iodixanol over other LOCMs on renal function have not been consistent $[9,10]$. Further conclusive comparisons are difficult due to highly variable definitions of CI-AKI and failure to account for confounding factors for AKI such as critical illness. Most studies also involved short-term follow-up and examined only acute renal dysfunction, while prognostication and knowledge of longer-term renal outcomes are scarce. 
Therefore, we wish to evaluate the incidence of CI-AKI and the temporal evolution of renal function over one year, in patients who received iodixanol versus iohexol for intraarterial angiography, using modern consensus definitions of AKI. We aim to identify the risk factors and prognostic implications of AKI in relation to different CM used, while accounting for confounding effects of CCF, CKD, and critical illness. We hypothesize that (i) use of iohexol versus iodixanol would be associated with a higher risk of CI-AKI and (ii) CI-AKI would, in turn, be associated with extended renal dysfunction (ERD) in medium-term survivors.

\section{Methods}

2.1. Study Design and Population. We performed a singlecentre, retrospective observational study, on the use of iodixanol (IOCM) versus iohexol (LOCM) in patients who underwent emergency or elective intra-arterial angiography, in our tertiary institution from January till December 2009. The patient list and identifiers were retrieved from routine procedural records. The Human Research Ethics Committee approved the study and waived the need for informed consent. All patients aged $>18$ years were included. The exclusion criteria were (i) patients with end-stage renal disease (ESRD) on maintenance dialysis, (ii) patients with no follow-up renal function assessment after angiography, and (iii) patients whose contrast type was not specified.

2.2. Study Definitions and Data Collection. All baseline patient demographics and comorbidities were indexed at time of angiography and captured in the procedural database. Critical illness was defined as admission(s) to the intensive care unit (ICU) within one week of angiography. The date of angiography was defined as day 0 (D0). Baseline serum creatinine level ( $\mathrm{sCr}$ ) was the patients' lowest $\mathrm{sCr}$ performed from D-7 to D0. If this was not available, it was inferred from the lowest $\mathrm{sCr}$ available in our electronic health records, up to one year from angiography. Baseline estimated glomerular filtration rate (eGFR) was calculated using the 4variable MDRD equation [11]. Peak sCr were obtained during respective time-windows from initial contrast exposure: D1D5, D20-D40, D70-D110, D150-D210, and D270-D450, as surrogates of renal function at 3 days, 30 days, 90 days, 6 months, and 1 year after angiography, respectively.

CI-AKI was defined as having fulfilled minimum " $R$ " criterion ( $\geq 1.5 \mathrm{x}$ increase in peak $\mathrm{sCr}$ from baseline) of the RIFLE “at-risk/injury/failure (R/I/F)" classification [12] at 3 days after contrast. ERD was described at medium-term (30 days and 90 days) and longer-term (6 months and 1 year), having to fulfil minimum RIFLE " $R$ " criterion at respective time-windows, which indicates failure to improve to baseline renal function. Corresponding delta-sCr levels were calculated from the difference between respective peak and baseline sCr.

2.3. Contrast and Angiography Details. We used iodixanol (Visipaque-320, GE Healthcare, Rydalmere, Australia) that contains $652 \mathrm{mg}$ iodixanol/mL and $320 \mathrm{mg}$ of elemental iodine and iohexol (Omnipaque-350, GE Healthcare, Rydalmere, Australia) that contains $755 \mathrm{mg}$ iohexol/ $\mathrm{mL}$ and $350 \mathrm{mg}$ of elemental iodine. The iodine dose was calculated from (iodine concentration of respective $\mathrm{CM} \times$ volume administered). The CM-load administered was defined as ratio of iodine dose infused corrected for baseline eGFR (grams iodine/eGFR), in view that iodine-dose to creatinineclearance ratio correlates well with the area under CM concentration-time curve [13].

The primary indication for angiography was identified from procedural records, including ST-elevation myocardial infarction (STEMI) and non-STEMI (NSTEMI). Likewise, angiography details including coronary angiogram (COROS), percutaneous coronary intervention (PCI), aortogram, left ventriculogram (LVgram), and intra-aortic balloon pump (IABP) insertion were retrieved if performed.

2.4. Data Analysis. The study subjects were divided into three cohorts for univariate analysis. Cohorts $\mathrm{A}, \mathrm{B}$, and $\mathrm{C}$ included all patients with available $\mathrm{sCr}$ at 3 days, 30 days, and 6 months, respectively. Incidences of CI-AKI and ERD were determined at prespecified time-points, between patients administered iodixanol and iohexol, and described separately for each cohort. Parametric variables were presented in mean ( \pm standard deviation) and nonparametric variables in median (interquartile range); univariate comparisons were then performed using Students' $t$-test and Wilcoxon ranksum test, respectively. Categorical variables were presented in frequency (percentage) and compared using Chi-square or Fisher-exact test.

For patients in cohort $A$, we examined the incidence of ERD over 1 year, between those with CI-AKI versus none. Subsequently, all clinically plausible variables in cohort $A$ were included in multivariate binary logistic and linear regression models, to look for independent predictors of CI$\mathrm{AKI} / \mathrm{ERD}$ and higher delta-sCr over one year, respectively. In particular, presence of CI-AKI and delta-sCr $>26 \mu \mathrm{mol} / \mathrm{L}$ at 3 days was included in the model for prediction of ERD. As delta-sCr was not of normal distribution, it was expressed as percentage change from baseline $\mathrm{sCr}$, with addition of a constant $(+100)$, and log-transformed prior to linear regression. The final models were developed using stepwise selection technique, with the $P$ value for inclusion being 0.05 . A two-sided $P<0.05$ was taken as measure of statistical significance. Finally, actuarial analysis was performed to illustrate the cumulative incidence of ERD with CI-AKI versus none, in cohort $A$ patients with one or more renal function assessments over subsequent one year, time/daycensored by the latest follow-up sCr measured. Analysis was performed using STATA SE version 13.0 (Lakeway Drive, College Station, Texas, USA).

\section{Results}

1229 patients underwent angiography and 713 patients fulfilled the study criteria, with 560, 190, and 172 patients in cohorts A, B, and $\mathrm{C}$, respectively. These cohorts were not 


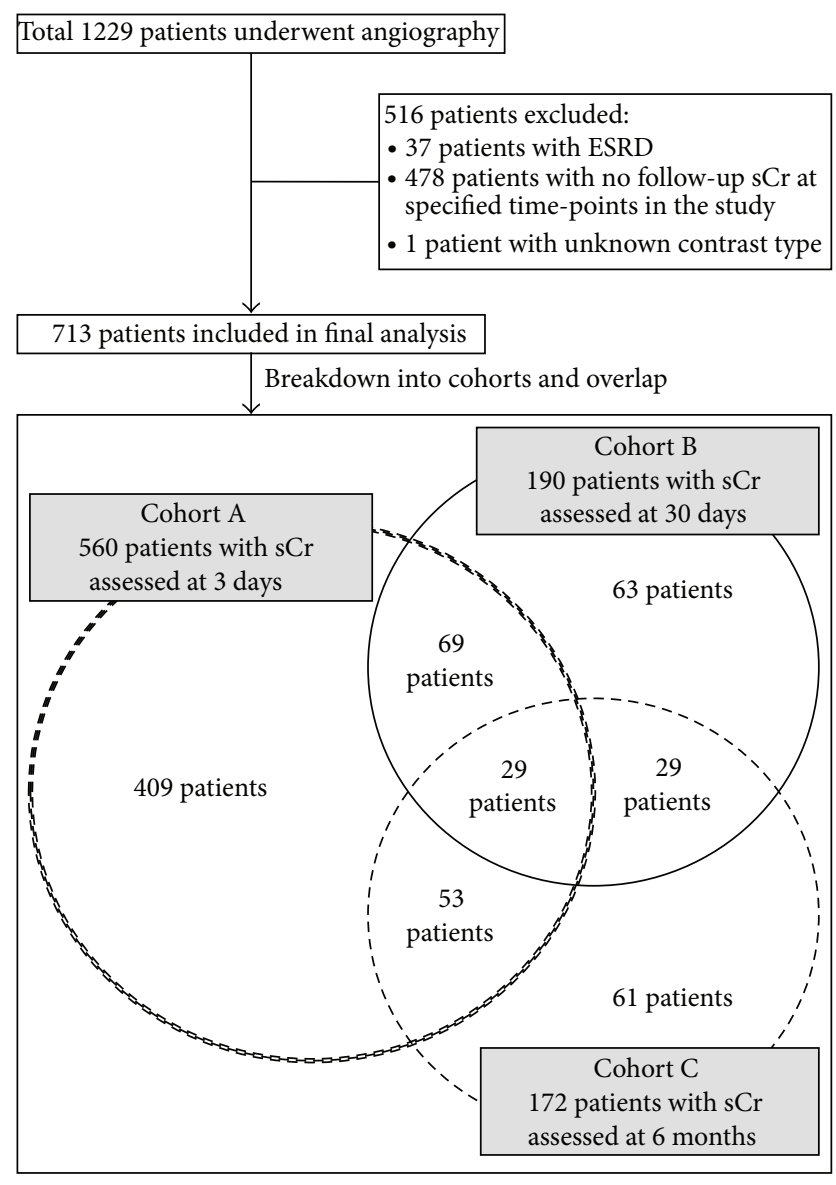

FIGURE 1: Study flow diagram. ESRD: end-stage renal disease; sCr: serum creatinine.

mutually exclusive (see Figure 1). Compared to 479 patients without ESRD who were excluded, these 713 patients had higher comorbid burden, and more underwent angiography and PCI for emergent indications (see Supplementary Material available online at http://dx.doi.org/10.1155/2014/ 506479).

Cohort A's profile is shown in Table 1, and profiles of cohorts $\mathrm{B}$ and $\mathrm{C}$ are shown in supplementary material. In general, patients who received iodixanol (versus iohexol) were older, and more had DM, CCF, STEMI (versus NSTEMI), and concomitant PCI, with higher CM load (corrected for eGFR) $(P<0.05)$. The baseline eGFR of patients given iodixanol versus iohexol in cohorts $\mathrm{A}, \mathrm{B}$, and $\mathrm{C}$ was $71( \pm 27)$ versus $88( \pm 26), 64( \pm 27)$ versus $96( \pm 36)$, and $65( \pm 27)$ versus $87( \pm 30) \mathrm{mL} / \mathrm{min} / 1.73 \mathrm{~m}^{2}$, respectively $(P<0.0001)$.

For cohort A, $18 \%$ had CI-AKI, which was more common in patients given iodixanol $(P=0.006)$. For cohort $\mathrm{B}, 32 \%$ had ERD at 30 days. For cohort C, 34\% had ERD at 6 months. 72 patients in cohort $\mathrm{C}$ had sCr assessed at 1 year, of which 29 patients (40\%) had longer-term ERD. The rates of mediumand longer-term ERD were comparable between patients who received iodixanol or iohexol at baseline.

More patients from cohort A with CI-AKI (compared with none) had ERD up to 6 months after angiography
$(P \leq 0.001)$ (Table 2). The corresponding incidences of ERD between the presence and absence of CI-AKI at 30 days, 90 days, and 6 months were $67 \%$ versus $26 \%, 75 \%$ versus $26 \%$, and $81 \%$ versus $26 \%$, respectively $(P \leq 0.001$ ) (see Supplementary Material). This association (actuarial analysis) is illustrated in Figure 2, in a subgroup of 209 patients from cohort A with one or more $\mathrm{sCr}$ levels assessed over subsequent one year.

On multivariate analysis (Table 3), baseline independent predictors of CI-AKI or higher delta-sCr (at 3-days) included age $>65$ years, female gender, CCF, STEMI, valvular heart disease or septal defect, IABP insertion, critical illness, PCI, and aortogram. Baseline eGFR $<45 \mathrm{~mL} / \mathrm{min} / 1.73 \mathrm{~m}^{2}$ and CM load $>0.7$ (per unit eGFR) were associated with lower delta$\mathrm{sCr}$ at 3 days. CI-AKI was consistently associated with both medium- and longer-term renal dysfunction. Delta-sCr $>26 \mu \mathrm{mol} / \mathrm{L}$ at 3 days was not associated with ERD. Type of $\mathrm{CM}$ (iodixanol or iohexol) was not independently associated with CI-AKI or ERD.

\section{Discussion}

4.1. Key Findings. The incidence of CI-AKI was $18 \%$. Advanced age, female gender, cardiac comorbidities, STEMI, and critical illness were key predictors of CI-AKI. Contrary to our first hypothesis, the association of CM type with CIAKI ceased to be significant after adjusting for the above confounders. In accordance with our second hypothesis, CIAKI was strongly associated with ERD over one year. ERD occurred in up to $30-40 \%$ of medium-term survivors who had follow-up renal assessment, and the risk was 3-fold higher in patients with CI-AKI.

4.2. Relationship with Previous Studies. CI-AKI is commonly defined by sCr rise $>0.5 \mathrm{mg} / \mathrm{dL}(44 \mu \mathrm{mol} / \mathrm{L})$ or $\geq 25 \%$ above baseline, and its reported incidence after angiography varies from $3 \%$ to $>20 \%$, depending on the population at risk [14-16]. Recent studies have classified CI-AKI using RIFLE criteria in patients who underwent coronary interventions. The RIFLE R/I/F incidence ranges from 8 to $16 \%$ [17, 18]. Our higher incidence of $18 \%$ can be attributed to our inclusion of cardiogenic shock or critical illness and our selected population in whom subsequent renal function assessments were necessary. By redefining CI-AKI from $\geq 25 \%$ to $\geq 50 \%$ rise in sCr using RIFLE criteria, we minimized the chance of overdiagnosis related to conventional definitions, since fluctuations in sCr may still occur in hospitalized patients who do not receive CM [19]. Such numerical fluctuations in sCr may be wider in advanced CKD, and thus defining CI-AKI by relative (versus absolute) change in sCr may be essential. A staging criterion also allows severity grading of CI-AKI. We report a combined RIFLE I/F ( $\geq 100 \%$ sCr rise) incidence of only $4 \%$, and historically only $<1 \%$ of patients had severe CI-AKI needing dialysis [20].

Risk predictors of CI-AKI such as advanced age and CCF are consistent with medical literature $[16,21]$. Our findings support the higher CI-AKI risk conferred by STEMI and need for IABP, which were previously noted in limited studies 
TABLE 1: Baseline profile and short-term impact on renal function (cohort A).

\begin{tabular}{|c|c|c|c|c|}
\hline Variables & $\begin{array}{l}\text { Cohort A } \\
n=560\end{array}$ & $\begin{array}{c}\text { Iodixanol (Visipaque) } \\
\qquad n=297\end{array}$ & $\begin{array}{l}\text { Iohexol (Omnipaque) } \\
\qquad n=263\end{array}$ & $P$ value \\
\hline Age, mean (SD), years & $65.1(12.1)$ & $67.4(12.0)$ & $62.5(11.8)$ & $<0.0001$ \\
\hline Age > 65 years, No. $(\%)$ & $282(50.4)$ & $177(59.6)$ & $105(39.9)$ & $<0.001$ \\
\hline Male gender, No. (\%) & $389(69.5)$ & $202(68.0)$ & $187(71.1)$ & 0.43 \\
\hline \multicolumn{5}{|l|}{ Comorbidities, No. (\%) } \\
\hline Diabetes mellitus & $93(16.6)$ & $60(20.2)$ & $33(12.6)$ & 0.02 \\
\hline Hypertensive heart disease & $337(60.2)$ & $184(62.0)$ & $153(58.2)$ & 0.36 \\
\hline CCF & $59(10.5)$ & $39(13.1)$ & $20(7.6)$ & 0.03 \\
\hline Critical illness (within 1 wk after contrast) & $53(9.5)$ & $30(10.1)$ & $23(8.8)$ & 0.58 \\
\hline \multicolumn{5}{|l|}{ Baseline renal function } \\
\hline Serum Cr, median (IQR), $\mu \mathrm{mol} / \mathrm{L}$ & $81(68-98)$ & $86(72-110)$ & $75(63-86)$ & $<0.0001$ \\
\hline $\mathrm{eGFR}^{*}$, mean $(\mathrm{SD}), \mathrm{mL} / \mathrm{min} / 1.73 \mathrm{~m}^{2}$ & $79(28)$ & $71(27)$ & $88(26)$ & $<0.0001$ \\
\hline $\mathrm{eGFR}^{*}<60 \mathrm{~mL} / \mathrm{min} / 1.73 \mathrm{~m}^{2}$, No. $(\%)$ & $138(24.6)$ & $110(37.0)$ & $28(10.7)$ & $<0.001$ \\
\hline $\mathrm{eGFR}^{*}<45 \mathrm{~mL} / \mathrm{min} / 1.73 \mathrm{~m}^{2}$, No. $(\%)$ & $57(10.2)$ & $46(15.5)$ & $11(4.2)$ & $<0.001$ \\
\hline \multicolumn{5}{|l|}{ Primary cardiac disease, No. (\%) } \\
\hline Suspect CAD (angina or CAD NOS) & $172(30.7)$ & $79(26.6)$ & $93(35.4)$ & 0.03 \\
\hline STEMI & $113(20.2)$ & $91(30.6)$ & $22(8.4)$ & $<0.001$ \\
\hline NSTEMI & $176(31.4)$ & $70(23.6)$ & $106(40.3)$ & $<0.001$ \\
\hline Arrhythmias & $26(4.6)$ & $12(4.0)$ & $14(5.3)$ & 0.47 \\
\hline Valvular heart disease/septal defects & $13(2.3)$ & $9(3.0)$ & $4(1.5)$ & 0.27 \\
\hline Cardiomyopathy & $17(3.0)$ & $11(3.7)$ & $6(2.3)$ & 0.33 \\
\hline Noncardiac issues & $9(1.6)$ & $7(2.4)$ & $2(0.8)$ & 0.18 \\
\hline Others & $34(6.1)$ & $18(6.1)$ & $16(6.1)$ & 0.99 \\
\hline \multicolumn{5}{|l|}{ Contrast load, median (IQR) } \\
\hline Contrast volume, $\mathrm{mL}$ & $150(100-230)$ & $160(100-240)$ & $145(100-210)$ & 0.80 \\
\hline Iodine content, g & $51(34-77)$ & $51(32-77)$ & $51(35-74)$ & 0.14 \\
\hline Iodine : eGFR ratio, g per $\mathrm{mL} / \mathrm{min} / 1.73 \mathrm{~m}^{2}$ & $0.66(0.44-1.02)$ & $0.73(0.47-1.13)$ & $0.60(0.42-0.91)$ & 0.006 \\
\hline Iodine : eGFR ratio $>0.7$ & $251(44.8)$ & $150(50.5)$ & $101(38.4)$ & 0.004 \\
\hline \multicolumn{5}{|l|}{ Procedure details, No. (\%) } \\
\hline Coronary angiogram & $551(98.4)$ & $291(98.0)$ & $260(98.9)$ & 0.51 \\
\hline PCI & $278(49.6)$ & $163(54.9)$ & $115(43.7)$ & 0.008 \\
\hline Aortogram & $34(6.1)$ & $17(5.7)$ & $17(6.5)$ & 0.71 \\
\hline LVgram & $365(65.2)$ & $191(64.3)$ & $174(66.2)$ & 0.65 \\
\hline IABP & $7(1.3)$ & $5(1.7)$ & $2(0.8)$ & 0.46 \\
\hline \multicolumn{5}{|l|}{ Renal function at 3 days after contrast } \\
\hline Peak sCr, median (IQR), $\mu \mathrm{mol} / \mathrm{L}$ & $87(73-106)$ & $96(81-121)$ & $78(67-90)$ & $<0.0001$ \\
\hline Median day of peak sCr & $1(1-2)$ & $1(1-2)$ & $1(1-2)$ & 0.13 \\
\hline$\Delta \mathrm{Cr}$, median (IQR), $\mu \mathrm{mol} / \mathrm{L}$ & $4(-2-16)$ & $8(-2-20)$ & $1(-3-10)$ & 0.001 \\
\hline RIFLE “R/I/F””, No. (\%) & $99(17.7)$ & $65(21.9)$ & $34(12.9)$ & 0.006 \\
\hline RIFLE “I/F”", No. (\%) & $21(3.8)$ & $14(4.7)$ & $7(2.7)$ & 0.20 \\
\hline
\end{tabular}

" 4-variable MDRD eGFR equation; " RIFLE acute kidney injury classification ("R/I/F” refers to "at-risk/injury/failure" classes, respectively).

$\Delta$ : delta (change in); CAD: coronary artery disease; CCF: congestive cardiac failure; D: day; eGFR: estimated glomerular filtration rate; IABP: intra-aortic balloon pump; IQR: interquartile range; LVgram: left ventriculogram; No.: number; NOS: not otherwise specified; NSTEMI: non-ST elevation myocardial infarction; PCI: percutaneous coronary intervention; pts: patients; sCr: serum creatinine; SD: standard deviation; STEMI: ST elevation myocardial infarction; wk: week.

$[22,23]$. These observations highlight the importance of circulatory failure, cardiogenic shock, and critical illness in influencing acute renal outcomes [24], which are potentially worsened by renal vasoconstriction following CM administration [25]. The inclusion of these emergent conditions in the multivariate model might have diminished the impact of baseline eGFR on CI-AKI. Patients with low baseline eGFR or at higher perceived risk of AKI might have been given prophylactic measures or lower doses of CM, hence explaining the reverse association of low eGFR and CM dose with CI-AKI. Furthermore, the results highlight the specific renal risk posed by STEMI, which is often accompanied 
TABLE 2: Extended renal outcomes in patients (cohort A) with CI-AKI versus none.

\begin{tabular}{|c|c|c|c|}
\hline Total 560 patients (cohort A) & $\begin{array}{c}\text { Non-CI-AKI } \\
n=461\end{array}$ & $\begin{array}{c}\text { CI-AKI } \\
n=99\end{array}$ & $P$ value \\
\hline Age $>65$ years, No. $(\%)$ & $218(47.3)$ & $64(64.7)$ & 0.002 \\
\hline Male gender, No. (\%) & $334(72.5)$ & $55(55.6)$ & 0.001 \\
\hline \multicolumn{4}{|l|}{ Comorbidities, No. (\%) } \\
\hline Diabetes mellitus & $70(15.2)$ & $23(23.2)$ & 0.05 \\
\hline Hypertensive heart disease & $281(61.0)$ & $56(56.6)$ & 0.42 \\
\hline $\mathrm{CCF}$ & $34(7.4)$ & $25(25.3)$ & $<0.001$ \\
\hline Critical illness (within 1 week after contrast) & $28(6.1)$ & $25(25.3)$ & $<0.001$ \\
\hline \multicolumn{4}{|l|}{ Baseline renal function } \\
\hline $\mathrm{eGFR}^{*}<60 \mathrm{~mL} / \mathrm{min} / 1.73 \mathrm{~m}^{2}$, No. (\%) & $120(26.0)$ & $18(18.2)$ & 0.10 \\
\hline $\mathrm{eGFR}^{*}<30 \mathrm{~mL} / \mathrm{min} / 1.73 \mathrm{~m}^{2}$, No. $(\%)$ & $45(9.8)$ & $12(12.1)$ & 0.48 \\
\hline Iohexol (vs iodixanol), No. (\%) & $229(49.7)$ & $34(34.3)$ & 0.006 \\
\hline Iodine dose per unit eGFR, median (IQR) & $0.68(0.45-1.04)$ & $0.60(0.40-0.89)$ & 0.06 \\
\hline Iodine dose per unit eGFR $>0.7$, No. $(\%)$ & $214(46.4)$ & $37(37.4)$ & 0.10 \\
\hline$\Delta \mathrm{Cr}$ at 3-days, median (IQR), $\mu \mathrm{mol} / \mathrm{L}$ & $0(-5-9)$ & $31(24-55)$ & $<0.0001$ \\
\hline \multicolumn{4}{|l|}{ Extended renal outcomes } \\
\hline At 6-months $(n=82)$ & $n=66$ & $n=16$ & \\
\hline$\Delta \mathrm{Cr}$, median (IQR), $\mu \mathrm{mol} / \mathrm{L}$ & $10(-1-27)$ & $39(25-45)$ & 0.0005 \\
\hline RIFLE “R/I/F”, No. (\%) & $17(25.8)$ & $13(81.3)$ & $<0.001$ \\
\hline RIFLE“I/F”\#, No. (\%) & $3(4.5)$ & $2(12.5)$ & 0.25 \\
\hline At 1-year $(n=80)$ & $n=72$ & $n=8$ & \\
\hline$\Delta \mathrm{Cr}, \operatorname{median}(\mathrm{IQR}), \mu \mathrm{mol} / \mathrm{L}$ & $9(-5-32)$ & $17(6-43)$ & 0.23 \\
\hline RIFLE “R/I/F”\#, No. (\%) & $21(29.2)$ & $4(50.0)$ & 0.25 \\
\hline RIFLE “I/F”\#, No. (\%) & $7(9.7)$ & $2(25.0)$ & 0.22 \\
\hline
\end{tabular}

${ }^{*} 4$-variable MDRD eGFR equation; ${ }^{\#}$ RIFLE acute kidney injury classification ("R/I/F” refers to "at-risk/injury/failure" classes, respectively).

$\Delta$ : delta (change in); eGFR: estimated glomerular filtration rate; IQR: interquartile range; No.: number; pts: patients; sCr: serum creatinine; SD: standard deviation; vs: versus.

by emergent revascularization and hemodynamic disturbance.

In vivo studies have demonstrated that $\mathrm{CM}$ induces renal tubular epithelial cell apoptosis [26]. Hyperosmolar solutions contribute to this cytotoxicity, and HOCM induces more renal tubular cell injury than LOCM [27]. In contrast, risk of cytotoxicity from IOCM or LOCM is similar [28], and there is no difference in clinically evident CI-AKI between iodixanol and different LOCMs from recent randomized studies [9, 10, 29]. A higher renal-risk posed by LOCM over IOCM seems to be confined to iohexol in patients with more advanced CKD [8]. In our model, choice of iohexol over iodixanol, or increasing CM load, did not strongly influence early or longer-term renal outcomes, in the presence of other stronger predictors of AKI. These findings support the notion that CI-AKI risk cannot be attributed to contrast osmolarity alone, and other mechanisms such as the clinical context, comorbidities, and hemodynamic condition may play a greater role in AKI pathogenesis.

Using similar modern consensus definitions, $28 \%$ of patients from the Alberta registry with mild CI-AKI $(\geq 50 \%$ or $\geq 0.3 \mathrm{mg} / \mathrm{dL}$ sCr rise) and $59 \%$ with more severe CI-AKI ( $\geq 100 \%$ sCr rise) had sustained AKI at 3 months, followed by a 0.8 and $2.8 \mathrm{~mL} / \mathrm{min} / 1.73 \mathrm{~m}^{2} /$ year decline in eGFR, respectively [30]. We report that $75 \%$ and $81 \%$ of patients with CI-AKI had ERD at 3 and 6 months, respectively, in a subcohort of at-risk patients with follow-up sCr measured

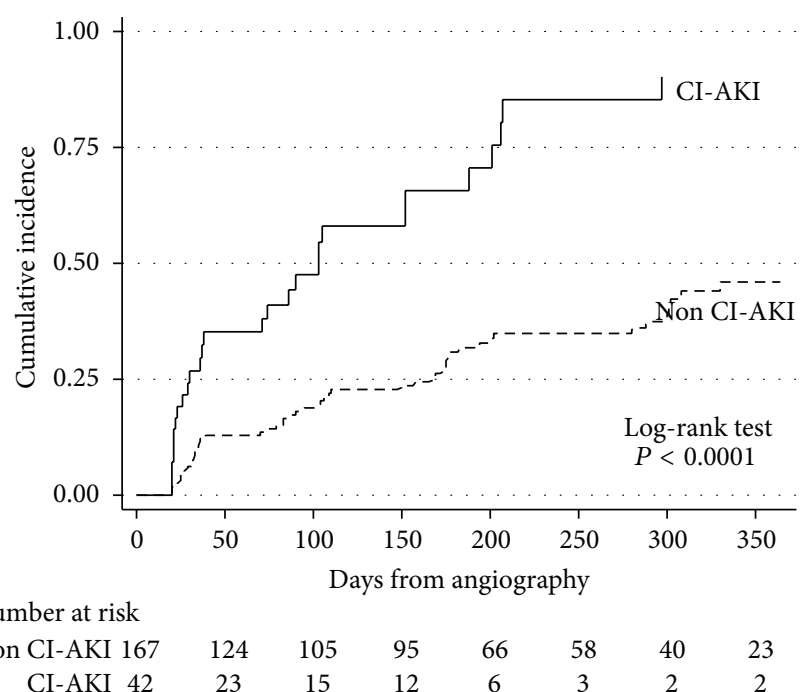

FIGURE 2: Actuarial analysis of extended renal dysfunction-CIAKI versus none. CI-AKI: contrast-induced acute kidney injury, defined by RIFLE R/I/F criteria at 3 days after contrast; R/I/F: atrisk/injury/failure; No.: number.

for clinical indications. More importantly, these patients with CI-AKI, especially those with ERD over months, have a significantly higher risk of long-term mortality, major 
TABLE 3: Multivariate analysis of renal dysfunction over one year after angiography (cohort A).

\begin{tabular}{|c|c|c|c|c|c|c|}
\hline \multirow[t]{2}{*}{ Significant variables } & \multicolumn{3}{|c|}{$\begin{array}{c}\text { Multivariate linear regression } \\
\text { (log-transformed } \% \Delta \mathrm{Cr}+\text { constant } \\
\left.\text { as dependent variable }{ }^{\dagger}\right)\end{array}$} & \multicolumn{3}{|c|}{$\begin{array}{c}\text { Multivariate logistic regression } \\
\text { (RIFLE “R/I/F” as dependent variable) }\end{array}$} \\
\hline & Coefficient & $95 \% \mathrm{CI}$ & $P$ value & Odds ratio & $95 \% \mathrm{CI}$ & $P$ value \\
\hline \multicolumn{7}{|l|}{ Renal dysfunction at 3 days after contrast } \\
\hline Age > 65 yrs $(\mathrm{Y} / \mathrm{N})$ & 0.05 & $0.01-0.09$ & 0.02 & 1.88 & $1.11-3.21$ & 0.02 \\
\hline Male gender (vs female) & -0.05 & $-0.09--0.01$ & 0.02 & 0.40 & $0.24-0.67$ & 0.001 \\
\hline $\mathrm{CCF}(\mathrm{Y} / \mathrm{N})$ & 0.07 & $0.01-0.14$ & 0.03 & 2.62 & $1.35-5.08$ & 0.004 \\
\hline Baseline eGFR < 45 & -0.09 & $-0.15--0.02$ & 0.009 & & & \\
\hline STEMI (Y/N) & 0.07 & $0.02-0.12$ & 0.007 & 2.08 & $1.11-3.89$ & 0.02 \\
\hline Valvular HD or septal defect $(\mathrm{Y} / \mathrm{N})$ & 0.14 & $0.02-0.27$ & 0.03 & 7.67 & $2.18-27.04$ & 0.002 \\
\hline Critical illness $(\mathrm{Y} / \mathrm{N})$ & 0.21 & $0.14-0.27$ & $<0.001$ & 4.45 & $2.18-9.08$ & $<0.001$ \\
\hline $\operatorname{IABP}(\mathrm{Y} / \mathrm{N})$ & 0.43 & $0.25-0.62$ & $<0.001$ & 17.66 & $1.80-173.55$ & 0.01 \\
\hline $\mathrm{PCI}(\mathrm{Y} / \mathrm{N})$ & 0.05 & $0.00-0.09$ & 0.04 & & & \\
\hline Aortogram (Y/N) & 0.08 & $0.00-0.16$ & 0.04 & & & \\
\hline Iodine dose per unit eGFR $>0.7$ & -0.08 & $-0.12--0.04$ & $<0.001$ & 0.50 & $0.29-0.86$ & 0.01 \\
\hline \multicolumn{7}{|c|}{ Renal dysfunction at 30 days after contrast ${ }^{*}$} \\
\hline CI-AKI (Y/N) & 0.30 & $0.13-0.47$ & 0.001 & 12.75 & $2.31-70.50$ & 0.004 \\
\hline Baseline eGFR < 45 & & & & 0.05 & $0.00-0.80$ & 0.04 \\
\hline Hypertensive HD (Y/N) & 0.16 & $0.01-0.30$ & 0.03 & 3.82 & $1.05-13.97$ & 0.04 \\
\hline Valvular HD or septal defect $(\mathrm{Y} / \mathrm{N})$ & 0.30 & $0.04-0.57$ & 0.03 & & & \\
\hline Aortogram (Y/N) & & & & 16.45 & $1.81-149.51$ & 0.01 \\
\hline Iodine dose per unit eGFR $>0.7$ & -0.20 & $-0.34--0.06$ & 0.007 & 0.08 & $0.02-0.33$ & 0.001 \\
\hline \multicolumn{7}{|c|}{ Renal dysfunction at 6 months after contrast* } \\
\hline CI-AKI (Y/N) & 0.39 & $0.26-0.52$ & $<0.001$ & 15.31 & $3.03-77.35$ & 0.001 \\
\hline Suspect CAD without AMI (Y/N) & 0.18 & $0.06-0.30$ & 0.003 & & & \\
\hline $\operatorname{IABP}(\mathrm{Y} / \mathrm{N})$ & -0.74 & $-1.24--0.25$ & 0.004 & & & \\
\hline $\mathrm{PCI}(\mathrm{Y} / \mathrm{N})$ & -0.18 & $-0.30--0.07$ & 0.003 & & & \\
\hline Aortogram $(\mathrm{Y} / \mathrm{N})$ & 0.25 & $0.05-0.45$ & 0.01 & & & \\
\hline \multicolumn{7}{|l|}{ Renal dysfunction at 1 year after contrast ${ }^{*}$} \\
\hline CI-AKI (Y/N) & 0.28 & $0.02-0.53$ & 0.03 & & & \\
\hline
\end{tabular}

Variables included in multivariate models include baseline variables: age > $65 \mathrm{yrs}$; male gender (vs females); DM (y/n); hypertensive HD (y/n); CCF (y/n); baseline eGFR $<45 \mathrm{~mL} / \mathrm{min} / 1.73 \mathrm{~m}^{2}$; suspect CAD without AMI $(\mathrm{y} / \mathrm{n}) ;$ STEMI $(\mathrm{y} / \mathrm{n}) ;$ NSTEMI $(\mathrm{y} / \mathrm{n}) ;$ arrhythmias $(\mathrm{y} / \mathrm{n})$; valvular HD or septal defect $(\mathrm{y} / \mathrm{n})$; $\operatorname{CMP}(\mathrm{y} / \mathrm{n})$ and periprocedure variables: COROS $(\mathrm{y} / \mathrm{n})$; aortogram $(\mathrm{y} / \mathrm{n}) ;$ PCI $(\mathrm{y} / \mathrm{n}) ; \operatorname{LVgram}(\mathrm{y} / \mathrm{n}) ; \operatorname{IABP}(\mathrm{y} / \mathrm{n})$; iohexol use (vs iodixanol); iodine dose per unit eGFR > 0.7; critical illness within $1 \mathrm{wk}$ after contrast $(\mathrm{y} / \mathrm{n})$.

${ }^{*}$ Additional variables added to model: CI-AKI $(\mathrm{y} / \mathrm{n}) ; \Delta \mathrm{Cr}>26 \mu \mathrm{mol} / \mathrm{L}(0.3 \mathrm{mg} / \mathrm{dL})$ at 3 days after contrast.

${ }^{\dagger}$ Delta-Cr expressed as \% change from baseline $\mathrm{Cr}$, with addition of constant (100), and log-transformed prior to linear regression.

$\triangle \mathrm{Cr}$ : (delta) change in Cr; AMI: acute myocardial infarction; CAD: coronary artery disease; CCF: congestive cardiac failure; CI: confidence interval; CMP: cardiomyopathy; COROS: coronary angiogram; Cr: serum creatinine; DM: diabetes mellitus; eGFR: estimated glomerular filtration rate; HD: heart disease; IABP: intra-aortic balloon pump; LVgram: left ventriculogram; NSTEMI: non-STEMI; PCI: percutaneous coronary intervention; STEMI: ST elevation myocardial infarction; vs: versus; wk: week; yrs: years.

cardiovascular events, and end-stage renal disease [31, 32]. These observations indicate that CI-AKI may reflect a higher propensity for recurrent renal dysfunction and adverse outcomes. Failure of AKI resolution over time might also suggest alternative disease mechanisms such as cholesterol embolism complicating acute illness and angiography [33].

4.3. Clinical Significance of Findings. Our study contributes to growing evidence that CI-AKI is not merely a transient and benign nephropathy but may reflect greater cardiovascular disease burden in high-risk patients [34]. We have identified CI-AKI as a major risk factor for progressive renal deterioration, and this should promote more assiduous follow-up and avoidance of nephrotoxins in these patients. The fact that only a minority of CI-AKI cases fulfilled the more severe RIFLE I/F criteria may be reassuring to clinicians. Finally, the understanding that CM type (iodixanol or iohexol) has less impact on acute or chronic renal outcomes in comparison to more important clinical variables might influence clinicians to reconsider their choice (or avoidance) of respective CM.

4.4. Strengths and Limitations. We have evaluated not just acute but extended renal outcomes over one year, in relation 
to multiple key clinical and procedural factors. The contribution of emergent coronary intervention and critical illness to CI-AKI had so far been assessed by limited studies, and we have demonstrated that these factors are more crucial than CM types in influencing clinical outcomes. This makes our study relevant to daily acute nephrology, cardiology, and radiology practice. We used the RIFLE criteria to classify CI-AKI, an established consensus system, well validated against patient outcomes. The impact of CI-AKI on ERD was strong, and key predictors of renal dysfunction appear logical, plausible, and consistent with expectations. However, iodixanol was used preferentially (over iohexol) in patients perceived at higher risk of AKI due to comorbidities including DM, CCF, CKD, and STEMI; patients with follow-up sCr measurements were naturally selected as individuals at-risk. These constitute selection bias, and the latter implies overinflated ERD incidence over time. But these mirror actual clinical practice and the results should remain relevant to physicians. Our study was single-centre and observational in nature and all associations cannot be inferred to have causal relationships. We were unable to examine differential outcomes according to various stages of RIFLE criteria, due to low patient numbers with RIFLE I/F. The retrospective nature and variable time window of available $\mathrm{sCr}$ also make it impossible to examine the 48-hour sCr change required of the AKIN criteria, which might be more sensitive in AKI diagnosis. We also did not have details of CI-AKI needing dialysis that is otherwise of clinical importance.

\section{Conclusions}

CI-AKI after angiography was common in an elderly patient cohort with significant cardiovascular disease burden, but the majority of cases were mild. The use of iodixanol versus iohexol or CM load has very limited or no positive association with renal outcomes, compared with advanced age, emergent cardiac conditions, cardiogenic shock, and critical illness. CIAKI is strongly associated with extended renal dysfunction over one year. Our study calls for further prospective research on extended renal outcomes of patients with CI-AKI over several years.

\section{Disclosure}

The paper has been seen and approved by all authors and it is not under consideration for publication elsewhere in a similar form, in any language, except in abstract form.

\section{Conflict of Interests}

The authors declare that there is no conflict of interests regarding the publication of this paper.

\section{Acknowledgment}

The abstract was presented during the poster presentation at the 31st Critical Care Nephrology Meeting, held in Vicenza, Italy, in June 2013.

\section{References}

[1] K. Nash, A. Hafeez, and S. Hou, "Hospital-acquired renal insufficiency," American Journal of Kidney Diseases, vol. 39, no. 5, pp. 930-936, 2002.

[2] R. Mehran, E. D. Aymong, E. Nikolsky et al., "A simple risk score for prediction of contrast-induced nephropathy after percutaneous coronary intervention: development and initial validation," Journal of the American College of Cardiology, vol. 44, no. 7, pp. 1393-1399, 2004.

[3] U. Nyman, J. Björk, P. Aspelin, and G. Marenzi, "Contrast medium dose-to-GFR ratio: a measure of systemic exposure to predict contrast-induced nephropathy after percutaneous coronary intervention," Acta Radiologica, vol. 49, no. 6, pp. 658$667,2008$.

[4] C. Mueller, G. Buerkle, H. J. Buettner et al., "Prevention of contrast media-associated nephropathy: randomized comparison of 2 hydration regimens in 1620 patients undergoing coronary angioplasty," Archives of Internal Medicine, vol. 162, no. 3, pp. 329-336, 2002.

[5] G. Marenzi, E. Assanelli, I. Marana et al., "N-acetylcysteine and contrast-induced nephropathy in primary angioplasty," The New England Journal of Medicine, vol. 354, no. 26, pp. 27732782, 2006.

[6] B. J. Barrett and E. J. Carlisle, "Metaanalysis of the relative nephrotoxicity of high- and low-osmolality iodinated contrast media," Radiology, vol. 188, no. 1, pp. 171-178, 1993.

[7] P. Aspelin, P. Aubry, S. Fransson, R. Strasser, R. Willenbrock, and K. J. Berg, "Nephrotoxic effects in high-risk patients undergoing angiography," The New England Journal of Medicine, vol. 348, no. 6, pp. 491-499, 2003.

[8] M. C. Heinrich, L. Häberle, V. Müller, W. Bautz, and M. Uder, "Nephrotoxicity of iso-osmolar iodixanol compared with nonionic low-osmolar contrast media: meta-analysis of randomized controlled trials," Radiology, vol. 250, no. 1, pp. 68-86, 2009.

[9] W. Laskey, P. Aspelin, C. Davidson et al., "Nephrotoxicity of iodixanol versus iopamidol in patients with chronic kidney disease and diabetes mellitus undergoing coronary angiographic procedures," The American Heart Journal, vol. 158, no. 5, pp. 822.e3-828.e3, 2009.

[10] L. Bolognese, G. Falsini, C. Schwenke et al., "Impact of iso-osmolar versus low-osmolar contrast agents on contrastinduced nephropathy and tissue reperfusion in unselected patients with ST-segment elevation myocardial infarction undergoing primary percutaneous coronary intervention (from the Contrast Media and Nephrotoxicity Following primary Angioplasty for Acute Myocardial Infarction [CONTRASTAMI] trial)," American Journal of Cardiology, vol. 109, no. 1, pp. 67-74, 2012.

[11] A. S. Levey, T. Greene, J. W. Kusek et al., "A simplified equation to predict glomerular filtration rate from serum creatinine," Journal of the American Society of Nephrology, vol. 11, abstract $155 \mathrm{~A}, 2000$.

[12] R. Bellomo, C. Ronco, J. A. Kellum, R. L. Mehta, and P. Palevsky, "Acute renal failure-definition, outcome measures, animal models, fluid therapy and information technology needs: The second international consensus conference of the acute dialysis quality initiative (adqi) group," Critical Care, vol. 8, no. 4, pp. R204-R212, 2004

[13] P. F. Sherwin, R. Cambron, J. A. Johnson, and J. A. Pierro, "Contrast dose-to-creatinine clearance ratio as a potential indicator of risk for radiocontrast-induced nephropathy: correlation of 
$\mathrm{D} / \mathrm{CrCL}$ with area under the contrast concentration-time curve using iodixanol," Investigative Radiology, vol. 40, no. 9, pp. 598603, 2005.

[14] P. A. McCullough, R. Wolyn, L. L. Rocher, R. N. Levin, and W. W. O'Neill, "Acute renal failure after coronary intervention: incidence, risk factors, and relationship to mortality," American Journal of Medicine, vol. 103, no. 5, pp. 368-375, 1997.

[15] C. S. Rihal, S. C. Textor, D. E. Grill et al., "Incidence and prognostic importance of acute renal failure after percutaneous coronary intervention," Circulation, vol. 105, no. 19, pp. 22592264, 2002.

[16] P. A. McCullough, "Acute kidney injury with iodinated contrast," Critical Care Medicine, vol. 36, no. 4, pp. S204-S211, 2008.

[17] M. Pakfetrat, M. H. Nikoo, L. Malekmakan et al., "A comparison of sodium bicarbonate infusion versus normal saline infusion and its combination with oral acetazolamide for prevention of contrast-induced nephropathy: a randomized, double-blind trial," International Urology and Nephrology, vol. 41, no. 3, pp. 629-634, 2009.

[18] M. Pakfetrat, M. H. Nikoo, L. Malekmakan et al., "Risk factors for contrast-related acute kidney injury according to risk, injury, failure, loss, and end-stage criteria in patients with coronary interventions," Iranian Journal of Kidney Diseases, vol. 4, no. 2, pp. 116-122, 2010.

[19] J. H. Newhouse, D. Kho, Q. A. Rao, and J. Starren, "Frequency of serum creatinine changes in the absence of iodinated contrast material: implications for studies of contrast nephrotoxicity," The American Journal of Roentgenology, vol. 191, no. 2, pp. 376$382,2008$.

[20] T. M. Labounty, M. Shah, S. V. Raman, F. Y. Lin, D. S. Berman, and J. K. Min, "Within-hospital and 30-day outcomes in 107,994 patients undergoing invasive coronary angiography with different low-osmolar iodinated contrast media," The American Journal of Cardiology, vol. 109, no. 11, pp. 1594-1599, 2012.

[21] R. Mehran and E. Nikolsky, "Contrast-induced nephropathy: definition, epidemiology, and patients at risk," Kidney International, vol. 69, pp. S11-S15, 2006.

[22] G. Marenzi, G. Lauri, E. Assanelli et al., "Contrast-induced nephropathy in patients undergoing primary angioplasty for acute myocardial infarction," Journal of the American College of Cardiology, vol. 44, no. 9, pp. 1780-1785, 2004.

[23] E. Chong, K. K. Poh, S. Liang, C. Y. Soon, and H.-C. Tan, "Comparison of risks and clinical predictors of contrastinduced nephropathy in patients undergoing emergency versus nonemergency percutaneous coronary interventions," Journal of Interventional Cardiology, vol. 23, no. 5, pp. 451-459, 2010.

[24] H. Chua, N. Glassford, and R. Bellomo, "Acute kidney injury after cardiac arrest," Resuscitation, vol. 83, no. 6, pp. 721-727, 2012.

[25] G. R. Hetzel, P. May, M. Hollenbeck, A. Voiculescu, U. Mödder, and B. Grabensee, "Assessment of radiocontrast media induced renal vasoconstriction by color coded duplexsonography," Renal Failure, vol. 23, no. 1, pp. 77-83, 2001.

[26] C. Quintavalle, M. Brenca, F. de Micco et al., "In vivo and in vitro assessment of pathways involved in contrast mediainduced renal cells apoptosis," Cell Death and Disease, vol. 2, no. 5, article e155, 2011.

[27] S. Duan, X. Zhou, F. Liu et al., "Comparative cytotoxicity of high-osmolar and low-osmolar contrast media on HKCs in vitro," Journal of Nephrology, vol. 19, no. 6, pp. 717-724, 2006.
[28] G. Romano, C. Briguori, C. Quintavalle et al., “Contrast agents and renal cell apoptosis," European Heart Journal, vol. 29, no. 20, pp. 2569-2576, 2008.

[29] R. J. Solomon, M. K. Natarajan, S. Doucet et al., "Cardiac angiography in renally impaired patients (CARE) study: a randomized double-blind trial of contrast-induced nephropathy in patients with chronic kidney disease," Circulation, vol. 115, no. 25, pp. 3189-3196, 2007.

[30] M. T. James, W. A. Ghali, M. Tonelli et al., "Acute kidney injury following coronary angiography is associated with a long-term decline in kidney function," Kidney International, vol. 78, no. 8, pp. 803-809, 2010.

[31] R. J. Solomon, R. Mehran, M. K. Natarajan et al., "Contrastinduced nephropathy and long-term adverse events: cause and effect?" Clinical Journal of the American Society of Nephrology, vol. 4, no. 7, pp. 1162-1169, 2009.

[32] M. Maioli, A. Toso, M. Leoncini, M. Gallopin, N. Musilli, and F. Bellandi, "Persistent renal damage after contrast-induced acute kidney injury: incidence, evolution, risk factors, and prognosis," Circulation, vol. 125, no. 25, pp. 3099-3107, 2012.

[33] P. A. McCullough, A. Adam, C. R. Becker et al., "Epidemiology and prognostic implications of contrast-induced nephropathy," American Journal of Cardiology, vol. 98, no. 6, pp. 5K-13K, 2006.

[34] M. T. James, S. M. Samuel, M. A. Manning et al., "Contrastinduced acute kidney injury and risk of adverse clinical outcomes after coronary angiography: a systematic review and meta-analysis," Circulation: Cardiovascular Interventions, vol. 6, no. 1, pp. 37-43, 2013. 


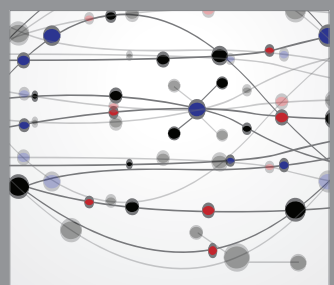

The Scientific World Journal
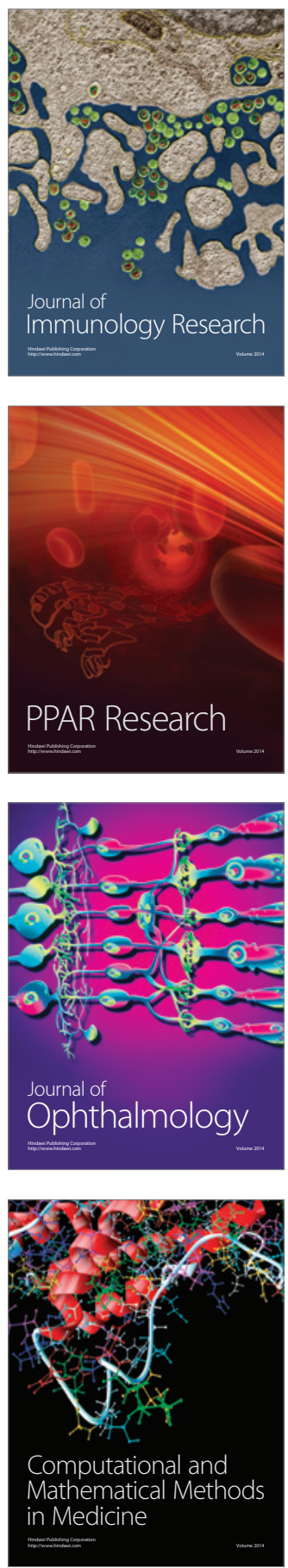

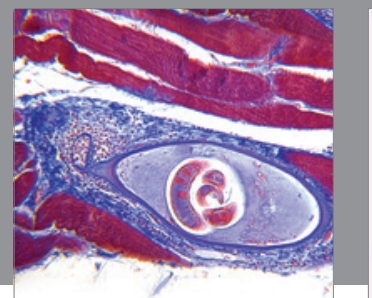

Gastroenterology

Research and Practice
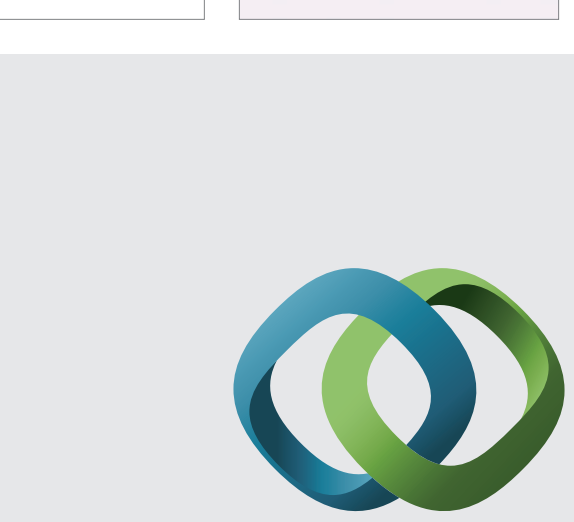

\section{Hindawi}

Submit your manuscripts at

http://www.hindawi.com
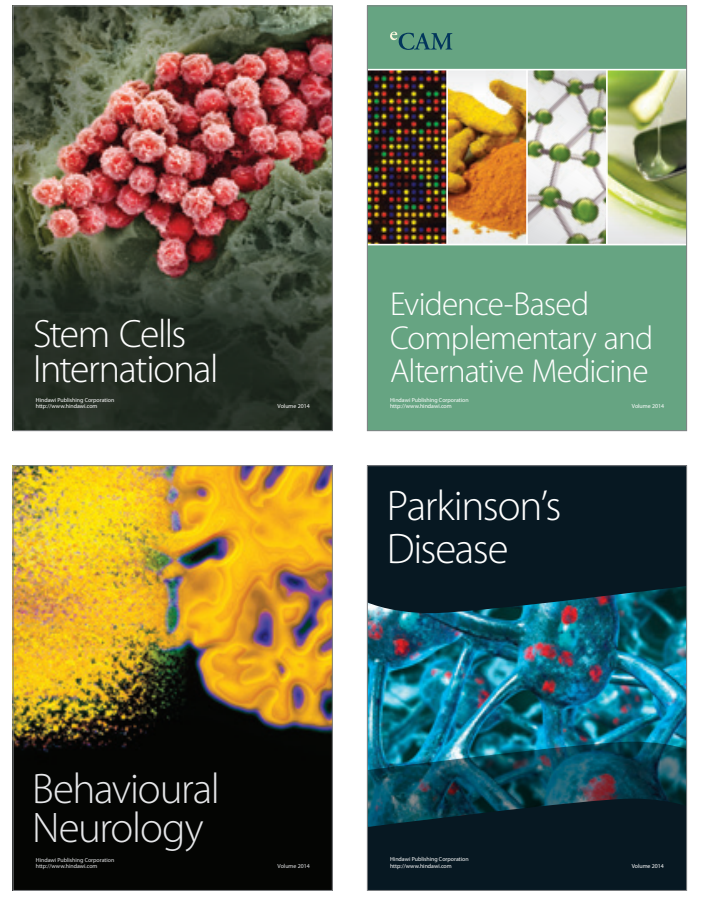
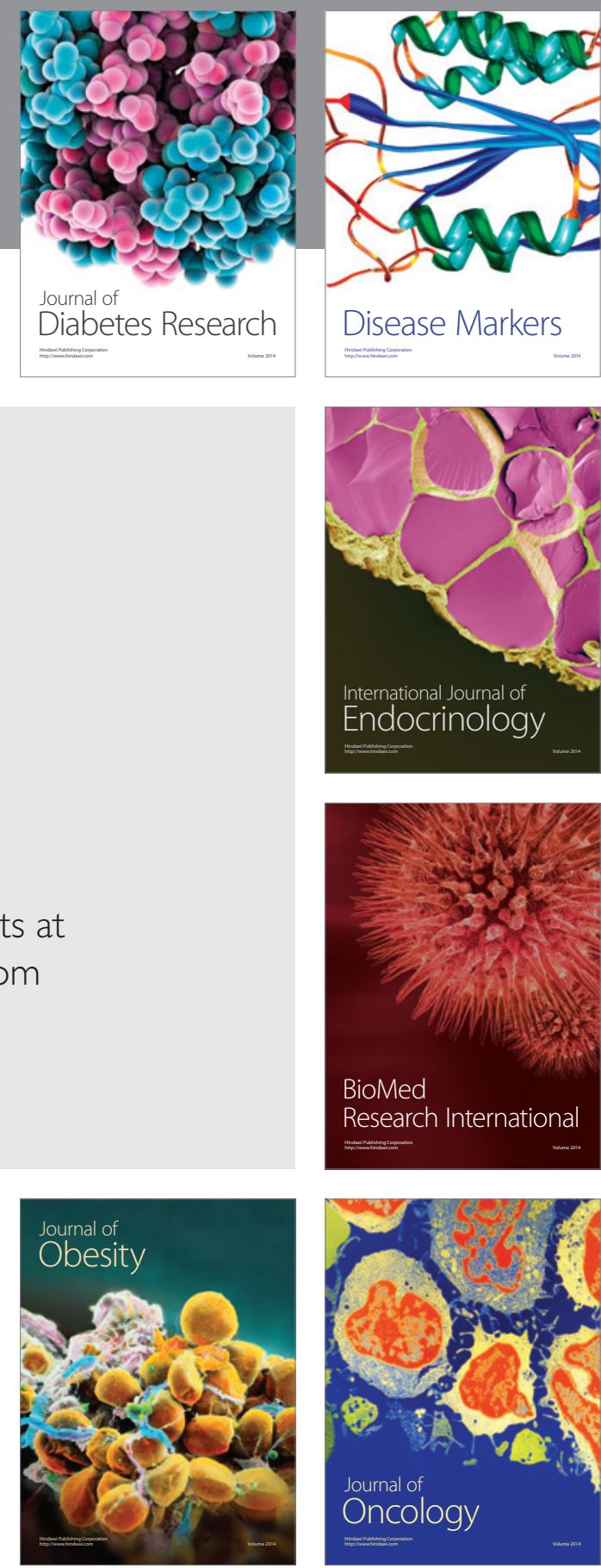

Disease Markers
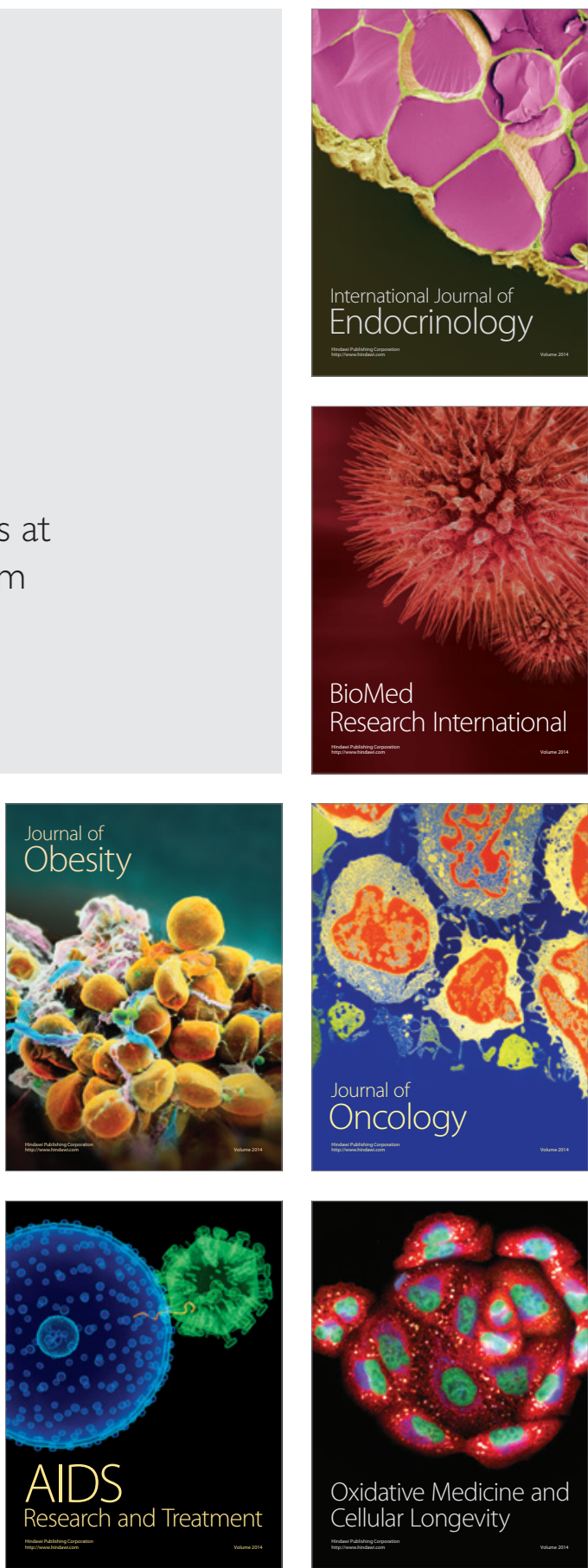